\title{
Matter and Radiation in Superstrong Magnetic Fields and Thermal Emission from Neutron Stars
}

\author{
Dong Lai and Wynn C.G. Ho \\ Department of Astronomy, Cornell University, Ithaca, NY 14853, USA
}

\begin{abstract}
Thermal surface emissions have now been detected from more than a dozen isolated neutron stars, including radio pulsars, radioquiet neutron stars and magnetars. These detections can potentially provide important information on the interior physics, magnetic fields, and surface composition neutron stars. Understanding the properties of matter and radiative transfer in strong magnetic fields is essential for the proper interpretation of the observations. We review current theoretical works on modeling magnetized neutron star atmospheres/surface layers, discussing some of the novel properties of matter and radiative transfer in strong magnetic fields. Of particular interest is the effect of the strong-field vacuum polarization, which dramatically changes the radiative transfer and the emergent X-ray spectra from magnetars.
\end{abstract}

\section{Introduction}

Neutron stars (NSs) are born in the core collapse and subsequent supernova explosion of massive stars and begin their lives at high temperatures, $T \gtrsim 10^{11} \mathrm{~K}$. As they cool over the next $10^{5}-10^{6}$ years, they act as sources of soft X-rays with surface temperatures $\gtrsim 10^{5} \mathrm{~K}$. The cooling history of the NS depends to a large extent on poorly constrained interior physics such as the nuclear equation of state, superfluidity, and magnetic field (see, e.g. Prakash et al. 2001; Yakovlev et a. 2001 for review). Already in 1960s, it was recognized that one way to study NSs is through observing their surface emissions in X-rays. This of course is very difficult because of the small NS size. Thus detecting NSs' surface radiation has been a long-term goal of high energy astrophysics for more than 30 years. Thanks to the advance in X-ray telescopes (starting from Einstein, but most notably ROSAT and the more recent ASCA, Chandra and XMM-Newton), such detection has become a reality. By now there are secured detections of surface emissions from more than a dozen isolated NSs, including radio pulsars (e.g., PSR B1055-52, B0656+14, Geminga, Vela), radio-quiet NSs (e.g., RX J1856.53754, RX J0720.4-3125), and magnetars (AXPs and SGRs) (see Becker \& Pavlov 2002 for a review). These detections are important because they probe the near vicinity and interior of NSs: From the spectrum we can measure the surface temperature, and thus constrain the NS radius and cooling history, which in turn depend on the interior physics; we can also potentially measure the surface 
composition and magnetic fields of $\mathrm{NSs}^{1}$. The thermal emission is mediated by the outmost layer of the NS. In order to properly interpret the current and future observations, it is crucial to have a detailed understanding of the physical properties of the matter and radiation in strong magnetic fields $\left(B \sim 10^{11_{-}}\right.$ $10^{16} \mathrm{G}$ ), and to calculate the emergent thermal radiation spectra from the NSs.

Some basics about NS atmospheres are in order. Because of the strong gravity, the NS atmosphere is highly compressed, with scale height $0.1-10 \mathrm{~cm}$ and density $\sim 0.1-10^{3} \mathrm{~g} / \mathrm{cm}^{3}$. Thus we are dealing with a highly nonideal gas and effects like pressure ionization are important. The physical properties of the atmosphere, such as the chemical composition, equation of state, and especially the radiative opacities, directly determine the characteristics of the thermal emission. While the surface composition of the NS is unknown, a great simplification arises due to the efficient gravitational separation of light and heavy elements. A pure $\mathrm{H}$ atmosphere is expected even if a small amount of fallback/accretion occurs after NS formation. A He atmosphere results if $\mathrm{H}$ is completely burnt up, and a heavy-element (e.g., C, O, or Fe) atmosphere may be possible if no fallback/accretion occurs. The strong magnetic field makes the atmospheric plasma anisotropic and birefringent. If the surface temperature is not too high, atoms and molecules may form in the atmosphere. Moreover, if the magnetic field is sufficiently strong, the NS envelope may transform into a condensed phase with very little gas above it. A superstrong magnetic field will also make some QED effects (e.g., vacuum polarization) important in calculating the surface radiation spectrum.

\section{Matter in Strong Magnetic Fields: Brief Overview}

We now briefly review the basic properties of matter in strong magnetic fields (see Lai 2001 and references therein).

An electron in a uniform magnetic field $B$ gyrates around the field line at the frequency $\omega_{c e}=e B /\left(m_{e} c\right)$. In quantum mechanics, this transverse motion is quantized into Landau levels, with the cyclotron energy (the Landau level spacing) given by $\hbar \omega_{c e}=11.58 B_{12} \mathrm{keV}$, and the cyclotron radius (the characteristic size of the wave packet) becomes $\hat{R}=(\hbar c / e B)^{1 / 2}=2.57 \times 10^{-10} B_{12}^{-1 / 2} \mathrm{~cm}$, where $B_{12}=B /\left(10^{12} \mathrm{G}\right)$. When studying matter in magnetic fields, the natural (atomic) unit for the field strength, $B_{0}$, is set by $\hbar \omega_{c e}=e^{2} / a_{0}$, where $a_{0}$ is the Bohr radius. Thus it is convenient to define a dimensionless magnetic field strength $b$ via

$$
b \equiv B / B_{0} ; \quad B_{0}=m_{e}^{2} e^{3} c / \hbar^{3}=2.3505 \times 10^{9} \mathrm{G} .
$$

For $b \gg 1$, the electron cyclotron energy $\hbar \omega_{c e}$ is much larger than the typical Coulomb energy, so that the properties of atoms, molecules and condensed matter are qualitatively changed by the magnetic field. In such a strong field regime,

\footnotetext{
${ }^{1}$ For example, the absorption features (around $0.7 \mathrm{keV}$ and $1.4 \mathrm{keV}$ ) detected in the radio-quiet NS 1E 1207-5209 by Chandra and XMM-Newton (Sanwal et al. 2002; Mereghetti et al. 2002; Hailey \& Mori 2002) have been interpreted as due to atomic level transitions of heavy elements in a $10^{11}-10^{12} \mathrm{G}$ field, or due to $\mathrm{He}^{+}$transitions in a $10^{14} \mathrm{G}$ field. However, the true nature of these lines is currently unclear, largely due to lack of theoretical modeling effort.
} 
the usual perturbative treatment of the magnetic effects (e.g., Zeeman splitting of atomic energy levels) does not apply. Instead, the Coulomb forces act as a perturbation to the magnetic forces, and the electrons in an atom/molecule settle into the ground Landau level. Because of the extreme confinement $\left(\hat{R} \ll a_{0}\right)$ of the electrons in the transverse direction (perpendicular to the field), the Coulomb force becomes much more effective in binding the electrons along the magnetic field direction. The atom attains a cylindrical structure. Moreover, it is possible for these elongated atoms to form molecular chains by covalent bonding along the field direction. Interactions between the linear chains can then lead to the formation of three-dimensional condensates.

We now discuss some basic properties of different bound states in strong magnetic fields, using $\mathrm{H}$ as an example.

(i) Atom: For $b \gg 1$, the $\mathrm{H}$ atom is elongated and squeezed, with the transverse size (perpendicular to $\mathbf{B}) \sim \hat{R}=a_{0} / b^{1 / 2} \ll a_{0}$ and the longitudinal size $\sim a_{0} /(\ln b)$. Thus the ground-state binding energy $|E| \simeq 0.16(\ln b)^{2}(\mathrm{au})$ (where $1 \mathrm{au}=27.2 \mathrm{eV}$; the factor 0.16 is an approximate number). Thus $|E|=$ $160,540 \mathrm{eV}$ at $B=10^{12}, 10^{14} \mathrm{G}$. In the ground state, the guiding center of the electron's gyro-motion coincides with the proton. The excited states of the atom can be obtained by displacing the guiding center away from the proton; this corresponds to $\hat{R} \rightarrow R_{s}=(2 s+1)^{1 / 2} \hat{R}$ (where $\left.s=0,1,2, \cdots\right)$. Thus $E_{s} \simeq-0.16\{\ln [b /(2 s+1)]\}^{2}(\mathrm{au})$.

This simple picture of the $\mathrm{H}$ energy levels is modified when we consider the effect of finite proton mass: Even for a "stationary" $\mathrm{H}$ atom, the energy $E_{s}$ is changed to $E_{s}+s \hbar \omega_{c p}$, where $\hbar \omega_{c p}=6.3 B_{12} \mathrm{eV}$ is the proton cyclotron energy. Thus the extra energy $s \hbar \omega_{c p}$ (which can be thought of as a "recoil" term) becomes increasingly important with increasing $B$. Moreover, the effect of center-of-mass motion is nontrivial: When the atom moves perpendicular to the magnetic field, a strong electric field is induced in its rest frame and can significantly change the atomic structure (the "motional Stark effect"); indeed, the mobility of the neutral atom across the magnetic field is limited. As a result, the dependence of the atomic energy on the transverse momentum is complicated. This effect leads to large shift and broadening of the energy levels and significant modification to the ionization equilibrium.

(ii) Molecules and Chains: In a strong magnetic field, the mechanism of forming molecules is quite different from the zero-field case. The spins of the electrons in the atoms are aligned anti-parallel to the magnetic field, and thus two atoms in their ground states do not bind together according to the exclusion principle. Instead, one $\mathrm{H}$ atom has to be excited to the $s=1$ state before combining (by covalent bond) with another atom in the $s=0$ state. Since the "activation energy" for exciting an electron in the $\mathrm{H}$ atom from $s$ to $(s+1)$ is small, the resulting $\mathrm{H}_{2}$ molecule is stable. Moreover, in strong magnetic fields, stable $\mathrm{H}_{3}, \mathrm{H}_{4}$ etc. can be formed in the similar manner. The dissociation energy of the molecule is much greater than the $B=0$ value: e.g., it is $40,350 \mathrm{eV}$ at $10^{12}, 10^{14} \mathrm{G}$ for $\mathrm{H}_{2}$, respectively.

(iii) Condensed Matter: As more atoms are added to a molecule, the energy per atom in a $\mathrm{H}_{n}$ molecule saturates, becoming independent of $n$; this occurs at $n \gtrsim\left[b /(\ln b)^{2}\right]^{1 / 5}(\sim 3-5$ for field strengths of interest). We then have a $1 \mathrm{D}$ metal. To obtain the basic scaling relation, we can consider a uniform 
cylinder model in which a $1 \mathrm{D}$ ion lattice is embedded in an electron Fermi sea. The radius of the cylinder and the ion spacing are of order $a \sim Z^{1 / 5} b^{-2 / 5}$ and the energy per "atom" is $E \sim-Z^{9 / 5} b^{2 / 5}$ (where we have restored the ion charge number $Z$ ). By placing parallel chains together (with spacing $\sim a$ ) we form a $3 \mathrm{D}$ condensed matter (e.g., in a body-centered tetragonal lattice). The energy per unit cell is again of order $E \sim-Z^{9 / 5} b^{2 / 5}$. The radius of the cell is $R \sim Z^{1 / 5} b^{-2 / 5}$, corresponding to the zero-pressure density $\simeq 10^{3} A Z^{3 / 5} B_{12}^{6 / 5} \mathrm{~g} \mathrm{~cm}^{-3}$ (where $A$ is the mass number of the ion).

(iv) Phase Diagram: Having understood the different bound states of $\mathrm{H}$, the next question is: What is the physical condition of the $\mathrm{H}$ surface layer of a NS as a function of $B$ and T? What is the phase diagram? Clearly, we are dealing with a highly magnetized, dense $\left(0.1-10^{3} \mathrm{~g} \mathrm{~cm}^{-3}\right)$ and partially ionized plasma; further complications arise from the strong coupling between the centerof-mass motion and the internal structure of atoms/molecules. Calculations indicate that there are two possible regimes: (1) Under "normal" conditions ( $B \lesssim 10^{14} \mathrm{G}$ and $T \gtrsim 10^{6} \mathrm{~K}$ ), the surface layer (photosphere) is gaseous and nondegenerate, with a mixture of $\mathrm{p}, \mathrm{e}, \mathrm{H}$ atoms, and $\mathrm{H}_{2}$, etc. (depending on $B, T, \rho$; (2) Under more "extreme" conditions $\left(T \gg 10^{14} \mathrm{G}\right.$ and/or $\left.T \ll 10^{6} \mathrm{~K}\right)$, there is a phase transition from the gaseous phase to the condensed metallic phase; as $B$ increases, the vapor density (above the metal) decreases, and we then have a situation in which the surface consists of condensed metallic $\mathrm{H}$ from which radiation directly comes out. The precise boundary between the two regimes (or the precise critical temperature) is currently quite uncertain.

\section{Neutron Star Atmosphere Modeling: Current Status}

Current zero-field NS atmospheres were based on the opacity and equation of state data from the OPAL project for pure $\mathrm{H}, \mathrm{He}$ and $\mathrm{Fe}$ compositions (Rajagopal \& Romani 1996; Zavlin et al. 1996; Gänsicke et al. 2002). These works showed that the radiation spectra from light-element $(\mathrm{H}$ or $\mathrm{He})$, low-field $\left(B \lesssim 10^{8} \mathrm{G}\right.$ ) atmospheres deviate significantly from blackbody. So far most studies of magnetic NS atmospheres have focused on $\mathrm{H}$ and moderate field strengths of $B \sim 10^{12}-10^{13} \mathrm{G}$ (see Zavlin \& Pavlov 2002 for a review). These models take into account the transport of different photon modes through a mostly ionized medium. The opacities adopted in the models include free-free transitions and electron scattering, while bound-free opacities are either neglected or treated in a very approximate manner and bound-bound transitions are ignored. Thus these magnetic atmosphere models are expected to be valid only for relatively high temperatures $\left(T \gtrsim\right.$ a few $\left.\times 10^{6} \mathrm{~K}\right)$ where hydrogen is almost completely ionized. As the magnetic field increases, we expect these models to break down at even higher temperatures as bound atoms, molecules and condensate become increasingly important. Models of magnetic iron atmospheres (with $B \sim 10^{12} \mathrm{G}$ ) were studied by Rajagopal et al. (1997). Because of the complexity in the atomic physics and radiative transport, these Fe models are necessarily crude. Despite some shortcomings, the $\mathrm{H}$ and $\mathrm{Fe}$ atmosphere models have played a valuable role in assessing the observed thermal spectra of NSs (see Pavlov et al. 2002 for a review). 

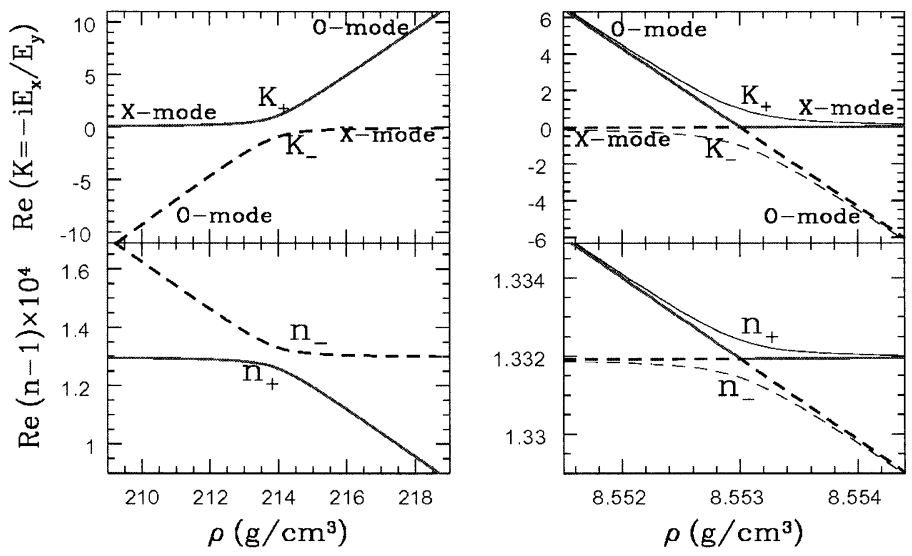

Figure 1. The polarization ellipticities $K$ (upper panels); and refractive indices (lower panels) of the photon modes as functions of density near vacuum resonance for $B=5 \times 10^{14} \mathrm{G}, \theta_{B}=45^{\circ}$, and $Y_{e}=1$. The left panels are for $E=5 \mathrm{keV}$, and the right panels for $E=1 \mathrm{keV}$. On the right panels, the light curves show the results when damping is neglected, while the heavy lines include damping (for $T=5 \times 10^{6} \mathrm{~K}$ ). On the left panels, the results are indistinguishable with and without damping. From Lai \& Ho (2003).

Much work remains to be done to incorporate bound species in magnetic NS atmosphere models. Although the atomic structure and radiative transitions of a atom in strong magnetic fields are well understood (e.g. Ruder et al. 1994; Mori et al. 2002), the difficulty arises from the effect of center-of-mass motion discussed in $\S 2$. Thermodynamically consistent equation of state and opacities for a magnetized $\mathrm{H}$ plasma have been calculated by Potekhin \& Chabrier (2003). Atmosphere models based on this equation of state shows that the most important transitions are from $s=0$ to $s=1$ and photoionization; both features are significantly broadened by the center-of-mass motion effect (see Ho et al. 2003).

The other area of recent development concerns NS atmospheres in the superstrong field regime $\left(B \gtrsim 10^{14} \mathrm{G}\right.$ ) (Ho \& Lai 2001, 2002, Lai \& Ho 2002, hereafter HL01,HL02,LH02; see also Özel 2001, Zane et al. 2001), which we discuss in $\S 4$.

\section{Radiative Transfer in Superstrong Magnetic Fields: Effect of Vac- uum Polarization}

Polarization of the vacuum due to virtual $e^{+} e^{-}$pairs becomes important when

$$
B \gtrsim B_{Q}=m_{e}^{2} c^{3} / e \hbar=4.414 \times 10^{13} \mathrm{G}
$$

this is a genuine quantum electrodynamics effect (e.g., Adler 1971; Heyl \& Hernquist 1997). Vacuum polarization modifies the dielectric property of the medium and the polarization of photon modes, thereby altering the radiative opacities 
(e.g., Gnedin et al. 1978; Meszaros \& Ventura 1979; Pavlov \& Gnedin 1984; Bulik \& Miller 1997; see Meszaros 1992 for a review). Of particular importance is the "vacuum resonance" phenomenon, which occurs when the effects of vacuum and plasma on the polarization of the modes "compensate" each other. For a photon of energy $E$, the vacuum resonance occurs at the density

$$
\rho_{V} \simeq 0.964 Y_{e}^{-1} B_{14}^{2} E_{1}^{2} f^{-2} \mathrm{~g} \mathrm{~cm}^{-3}
$$

where $Y_{e}$ is the electron fraction, $E_{1}=E /(1 \mathrm{keV}), B=10^{14} B_{14} \mathrm{G}$ is the magnetic field strength, and $f=f(B)$ is a slowly varying function of $B$ and is of order unity (LH02 and HL02). For $\rho>\rho_{V}$ (where the plasma effect dominates the dielectric tensor) and $\rho<\rho_{V}$ (where vacuum polarization dominates), the photon modes (for $E$ much smaller than the electron cyclotron energy $E_{B e}$ ) are almost linearly polarized (see Fig. 1): the extraordinary mode (X-mode) has its electric field vector $\mathbf{E}$ perpendicular to the $\hat{\mathbf{k}}-\hat{\mathbf{B}}$ plane, while the ordinary mode (O-mode) is polarized along the $\hat{\mathbf{k}}-\hat{\mathbf{B}}$ plane (where $\hat{\mathbf{k}}$ specifies the direction of photon propagation, $\hat{\mathbf{B}}$ is the unit vector along the magnetic field). Near $\rho=\rho_{V}$, however, the normal modes become circularly polarized as a result of the "cancellation" of the plasma and vacuum effects - both effects tend to make the mode linearly polarized, but in mutually orthogonal directions. When a photon propagates in an inhomogeneous medium, its polarization state will evolve adiabatically if the density variation is sufficiently gentle. Thus, a X-mode (O-mode) photon will be converted into a $\mathrm{O}$-mode (X-mode) as it traverses the vacuum resonance (see Fig. 2). This resonant mode conversion is analogous to the MSW effect of neutrino oscillation (e.g., Haxton 1995). For this conversion to be effective, the adiabatic condition must be satisfied:

$$
E \gtrsim E_{\mathrm{ad}}\left(B, \theta_{B}, H_{\rho}\right)=1.49\left(f \tan \theta_{B}\left|1-u_{i}\right|\right)^{2 / 3}\left(5 \mathrm{~cm} / H_{\rho}\right)^{1 / 3} \mathrm{keV},
$$

where $\theta_{B}$ is the angle between $\hat{\mathbf{k}}$ and $\hat{\mathbf{B}}, u_{i}=\left(E_{B i} / E\right)^{2}, E_{B i}$ is the ion cyclotron energy, and $H_{\rho}=|d z / d \ln \rho|$ is the density scale height (evaluated at $\rho=\rho_{V}$ ) along the ray. For an ionized Hydrogen atmosphere, $H_{\rho} \simeq 2 k T /\left(m_{p} g \cos \theta\right)=$ $1.65 T_{6} /\left(g_{14} \cos \theta\right) \mathrm{cm}$, where $T=10^{6} T_{6} \mathrm{~K}$ is the temperature, $g=10^{14} g_{14} \mathrm{~cm} \mathrm{~s}^{-2}$ is the gravitational acceleration, and $\theta$ is the angle between the ray and the surface normal. We note that here we refer to the O-mode as the mode with $|K|=\left|E_{x} / E_{y}\right| \gg 1$ and the X-mode as the mode with $|K| \ll 1$ (here the $z$-axis is along $\hat{\mathbf{k}}$ and the $y$-axis is in the direction of $\hat{\mathbf{B}} \times \hat{\mathbf{k}}$ ), thus the name "mode conversion". Alternatively, one can define modes with definite helicity (the sign of $K=-i E_{x} / E_{y}$ ) such that $K$ changes continuously as $\rho$ changes; we call these plus-mode and minus-mode. Thus we may also say that in the adiabatic limit, the photon will remain in the same plus or minus branch, but the character of the mode is changed across the vacuum resonance. Indeed, in the literature on radio wave propagation in plasmas, the nonadiabatic case, in which the photon state jumps across the continuous curves, is referred to as "linear mode coupling". It is important to note that the "mode conversion" effect discussed here is not a matter of semantics. The key point is that in the adiabatic limit, the 
photon polarization ellipse changes its orientation across the vacuum resonance, and therefore the photon opacity changes significantly ${ }^{2}$.
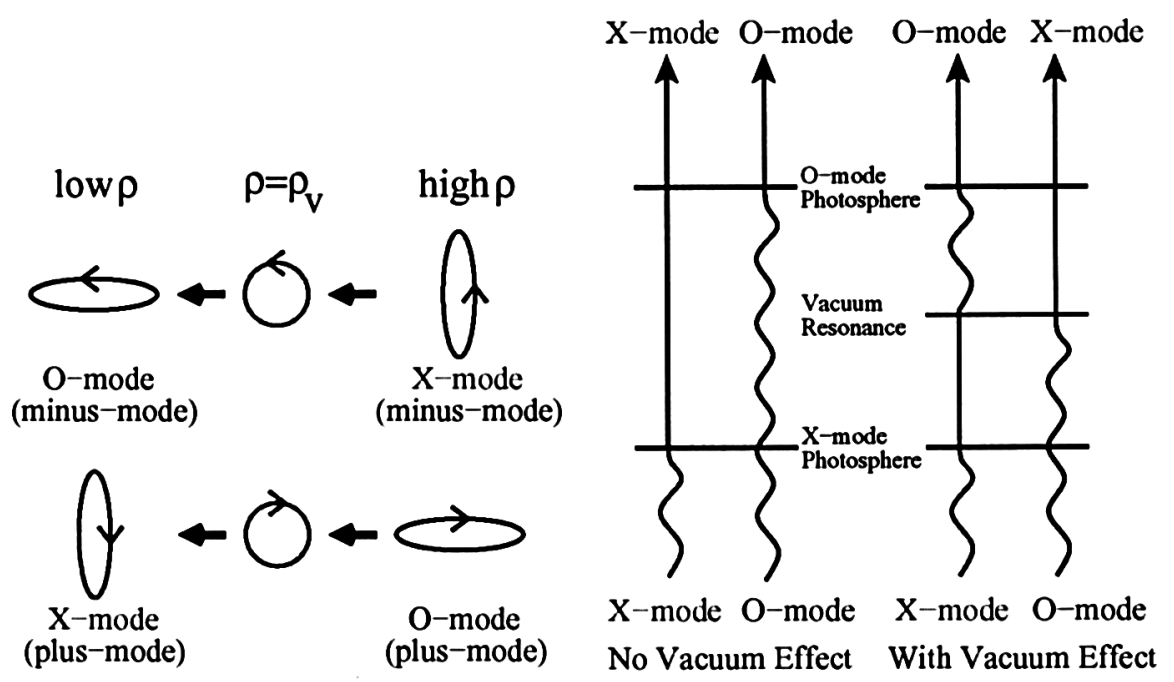

Figure 2. Left panel (a schematic diagram illustrating mode conversion due to vacuum polarization): As a minus-mode (plus-mode) photon, which manifests as X-mode (O-mode) at high density (for $\left.E>E_{B i}\right)$, traverses the vacuum resonance density $\rho_{V}$, it will stay as minus-mode (plus-mode) and become O-mode (X-mode) at low density if the adiabatic condition is satisfied; in this evolution, the polarization ellipse rotates $90^{\circ}$ and the photon opacity changes significantly. Right panel (a diagram illustrating how vacuum polarization-induced mode conversion affects radiative transfer in a magnetar atmosphere): When the vacuum polarization effect is turned off, the X-mode photosphere (where optical depth $\sim 1$ ) lies deeper than the O-mode; with the vacuum polarization effect included, the $\mathrm{X}$-mode effectively decouples (emerges) from the atmosphere at the vacuum resonance, which lies at a lower density than the (original) X-mode photosphere. From Lai \& Ho (2003).

Because the two photon modes have vastly different opacities (see footnote 2 ), the vacuum polarization-induced mode conversion can significantly affect radiative transfer in magnetar atmospheres. The main effect of vacuum polarization on the atmosphere spectrum can be understood as follows. When the vacuum polarization effect is neglected, the decoupling densities of the O-mode and X-mode photons (i.e., the densities of their respective photospheres) are approximately given by (for a $\mathrm{H}$ plasma) $\rho_{O} \simeq 0.42 T_{6}^{-1 / 4} E_{1}^{3 / 2} G^{-1 / 2} \mathrm{~g} \mathrm{~cm}^{-3}$ and

\footnotetext{
${ }^{2}$ The O-mode has a significant component of its $\mathbf{E}$ field along $\hat{\mathbf{B}}$ (for most directions of propagation except when $\hat{\mathbf{k}}$ is nearly parallel to $\hat{\mathbf{B}}$ ), and therefore the O-mode opacity is close to the $B=0$ value, while the $\mathrm{X}$-mode opacity is much smaller.
} 
$\rho_{X} \simeq 486 T_{6}^{-1 / 4} E_{1}^{1 / 2} B_{14} G^{-1 / 2} \mathrm{~g} \mathrm{~cm}^{-3}$ (see LH02), where $G=1-e^{-E / k T}$. The vacuum resonance lies between these two photospheres when $\rho_{O}<\rho_{V}<\rho_{X}$, i.e.

$$
0.66 f T_{6}^{-1 / 8} E_{1}^{-1 / 4} G^{-1 / 4}<B_{14}<510 f^{2} T_{6}^{-1 / 4} E_{1}^{-3 / 2} G^{-1 / 2} .
$$

When this condition is satisfied, the effective decoupling depths of the photons are changed ${ }^{3}$. Indeed, we see from Fig. 2 (right panel) that mode conversion makes the effective decoupling density of X-mode photons (which carry the bulk of the thermal energy) smaller, thereby depleting the high-energy tail of the spectrum and making the spectrum closer to black-body (although the spectrum is still harder than black-body because of nongrey opacities) ${ }^{4}$. This expectation is borne out in self-consistent atmosphere modeling presented in HL02 (see Fig. 3). Another important effect of vacuum polarization on the spectrum, first noted in HL02, is the suppression of proton cyclotron lines (and maybe other spectral lines; see Ho et al. 2003). The physical origin for such line suppression is related to the depletion of continuum flux, which makes the decoupling depths inside and outside the line similar. HL02 suggests that the absence of lines in the observed spectra of several AXPs (e.g., Juett et al. 2002; Tiengo et al. 2002) may be an indication of the vacuum polarization effect at work in these systems.

Our previous study (LH02) of the vacuum-induced mode conversion did not explicitly take into account of the effect of dissipation on the mode structure. Although this dissipative effect is small under many situations, near the vacuum resonance and for some directions of propagation, the two photon modes can "collapse", i.e., they become identical and hence nonorthogonal (Soffel et al. 1983). The analysis of LH02 breaks down near these "mode collapse" points. Also, all previous studies of NS atmospheres with strong magnetic fields rely on the modal description of the radiative transport. This is valid only in the limit of large Faraday depolarization (Gnedin \& Pavlov 1974), which is not always satisfied near the vacuum resonance, especially for superstrong field strengths (HL02). More importantly, the transfer equations based on normal modes cannot handle the cases in which partial mode coupling (conversion) occurs across the vacuum resonance (i.e., when the adiabatic condition is neither strongly satisfied or violated). Clearly, to properly account for the effects of mode collapse, breakdown of Faraday depolarization, and mode conversion associated with vacuum polarization, one must go beyond the modal description of the radiation field by formulating and solving the transfer equation in terms of the photon intensity matrix (or Stokes parameters) and including the birefringence of the plasma-vacuum medium (see Lai \& Ho 2003).

Acknowledgments. This work is supported in part by NASA grants NAG 5-8484 and NAG 5-12034, and NSF grant AST 9986740.

\footnotetext{
${ }^{3}$ For lager $B$ the vacuum resonance lies deeper than the photospheres of both modes, while for smaller $B$ the resonance lies outside both photospheres. In both cases, the effect of vacuum polarization on the radiative spectrum is expected to be small, although in the latter case (smaller $B$ ) the polarization of the emitted photons will be modified by the vacuum resonance.

${ }^{4}$ Even when mode conversion is neglected, the X-mode decoupling depth can still be affected by vacuum polarization. This is because the $\mathrm{X}$-mode opacity exhibits a spike feature near the resonance, and the optical depth across the resonance region can be significant; see LH02.
} 

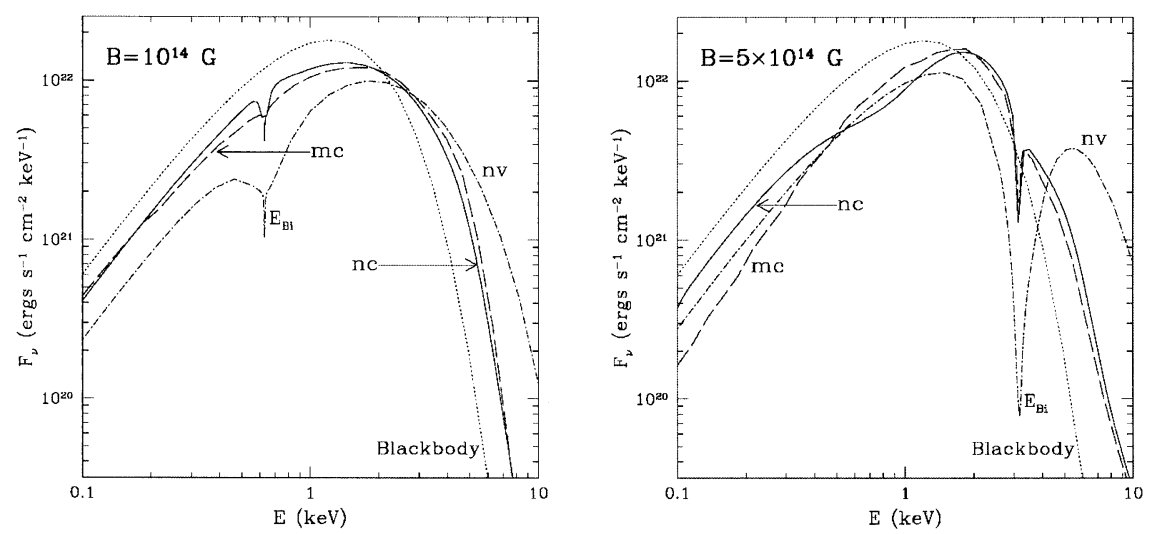

Figure 3. Spectra of fully ionized $\mathrm{H}$ atmospheres with $B_{14}=1$ (left) and 5 (right), both for $T_{\text {eff }}=5 \times 10^{6} \mathrm{~K}$, and $\mathbf{B}$ perpendicular to the surface. The solid line is for an atmosphere with vacuum polarization but no mode conversion (nc), the dashed line is for an atmosphere with complete vacuum-induced mode conversion $(\mathrm{mc})$, the dot-dashed line is for an atmosphere with no vacuum polarization (nv), and the dotted line is the blackbody spectrum. The $E_{B i}=0.63 B_{14} \mathrm{keV}$ features are due to ion cyclotron resonance. From HL02.

\section{References}

Adler, S.L., 1971, Ann. Phys., 67, 599

Becker, W., \& Pavlov, G.G. 2002, in The Century of Space Science, ed. J. Bleeker et al. (Kluwer) (astro-ph/0208356)

Bulik, T., \& Miller, M.C. 1997, MNRAS, 288, 596

Drake, J.J. et al. 2002, ApJ, 572, 996

Gänsicke, B. T., Braje, T. M., \& Romani, R.W. 2002, A\&A, 386, 1001

Gnedin, Yu.N. \& Pavlov, G.G. 1974, Sov. Phys. JETP, 38, 903

Gnedin, Yu.N., et al. 1978, Sov. Astron. Lett., 4, 117

Hailey, C.J., \& Mori, K. 2002, ApJ, 578, L133

Haxton, W.C. 1995, ARAA, 33, 459

Heyl, J.S. \& Hernquist, L., 1997, J. Phys. A30, 6485

Ho, W.C.G., \& Lai, D. 2001, MNRAS, 327, 1081

Ho, W.C.G., \& Lai, D. 2002, MNRAS, in press (astro-ph/0201380)

Ho, W.C.G., Lai, D., Potekhin, A., \& Chabrier, G. 2003, submitted 
Juett, A.M., et al. 2002, ApJ, 568, L31

Lai, D. 2001, "Matter in Strong Magnetic Fields", Rev. Mod. Phys., 73, 629

Lai, D., \& Ho, W.C.G. 2002, ApJ, 566, 373

Lai, D., \& Ho, W.C.G. 2003, ApJ, submitted

Mereghetti, S., et al. 2002, ApJ, in press

Mészáros, P. 1992, High Energy Radiation from Magnetized Neutron Stars (The Univ. of Chicago Press: Chicago)

Mészáros, P. \& Ventura, J. 1979, Phys. Rev. D19, 3565

Mori, K., \& Hailey, C.J. 2002, ApJ, 564, 914

Özel, F. 2001, ApJ, 563, 276

Pavlov, G.G., \& Gnedin, Y.N. 1984, Sov. Sci. Rev. E Astro. Space Phys., 3, 197

Pavlov, G.G., Zavlin, V.E., \& Sanwal, D. 2002, astro-ph/0206024

Potekhin, A.Y., \& Chabrier, G. 2003, ApJ, in press

Prakash, M., et al. 2001, in Physics of Neutron Star Interiors, eds. D. Blaschke, N.K. Glendenning \& A. Sedrakian (Springer) (astro-ph/0012136)

Rajagopal, M., \& Romani, R.W. 1996, ApJ, 461, 327

Rajagopal, M., Romani, R.W., \& Miller, M.C. 1997, ApJ, 479, 347

Ruder, H. et al. 1994, Atoms in Strong Magnetic Fields (Springer-Verlag)

Sanwal, D., et al. 2002, ApJ, 574, L61

Soffel, M., et al. 1983, A\&A, 126, 251

Tiengo, A., Goehler, E., Staubert, R., \& Mereghetti, S. 2002, A\&A, 383, 182

Yakovlev, D.G., et al. 2001, Phys. Rep., 354, 1 (astro-ph/0012122)

Zane, S., Turolla, R., Stella, L., \& Treves, A. 2001, ApJ, 560, 384

Zavlin, V.E. \& Pavlov, G.G. 2002, in Proc. 270 WE-Heraeus Seminar on Neutron Stars, Pulsars, and Supernova Remnants, eds. W. Becker et al

Zavlin, V.E., Pavlov, G.G., \& Shibanov, Y.A. 1996, A\&A, 315, 141 